Full-Length Article

\title{
Clinical observations of live improvisational harp music in neonatal intensive care
}

Roxanne McLeod ${ }^{1}$, Kaye Spence ${ }^{2,3}$

${ }^{1}$ The Children's Hospital at Westmead, part of the Sydney Children's Hospitals Network, Sydney, Australia

${ }^{2}$ Grace Centre for Newborn Intensive Care

${ }^{3}$ School of Nursing and Midwifery, Western Sydney University

\begin{abstract}
Music Therapy services in Neonatal Intensive Care Units has received growing attention through an increasing body of research and practice. As part of a developmental program in a Neonatal Intensive Care Unit, live improvisational harp music was used with a selection of medically stable infants and their families. The music intervention used a Reverie Harp played by a qualified music therapist, tailored to the infant's individual responses. These sessions were simultaneously observed by a nurse who was certified in NIDCAP (Newborn Individualized Developmental Care and Assessment Program). The NIDCAP observations were used to note what effect, if any, this type of music intervention had on the infant's behavioral state and regulation. The behavioral observations and the responses to the music were shown to be calming and enabled the infants to self-regulate their behavior. Further research is required to substantiate the efficacy of live improvisational harp music tailored to the infant's responses and its place among other music interventions in the Neonatal Intensive Care Unit. Collaborating with an experienced nurse in the introduction of music therapy services is worthwhile in identifying infants and their families who may benefit from specific music interventions.
\end{abstract}

Keywords: NICU, music therapy, live music, Reverie Harp, NIDCAP, Neonate

multilingual abstract $\mid$ mmd.iammonline.com

\section{Introduction}

The delivery of Music Therapy (MT) services in the Neonatal Intensive Care Unit (NICU) has received growing attention through an increasing body of research into effectiveness with pacification, infant development, pain reduction, and attachment with primary caregivers $[1,2,3,4,5]$. Demonstrated benefits of MT interventions for infants include influencing cardiac and respiratory function [2,6,7]; quicker settling after heel lance procedures [8,9,10]; reducing frequency and duration of inconsolable crying [11]; improved physiological states and reduction of stress-related behaviors [6,7,12]; increased weight gain $[13,14]$ and improvements in nonnutritive sucking behaviors $[7,15]$. The neurobiological benefits of music in early intervention have also been noted $[16,17]$. The benefits of MT interventions have been demonstrated to also extend to parents in the NICU including a reduction of parental anxiety $[2,14,18,19]$, facilitating

PRODUCTION NOTES: Address correspondence to:

Roxanne McLeod BSocSc, MAMusTher | E-mail: roxanne.mcleod@health.nsw.gov.au | Address: The Children's Hospital at Westmead. Locked Bag 4001, Westmead, NSW 2145, Australia| COI statement: The authors declared that no financial support was given for the writing of this article. The authors have no conflict of interest to declare. bonding with infants $[5,7,20]$, and providing parents with an active role in promoting their child's recovery and growth $[19,21]$.

The use of pentatonic harps and lyres in developmental care for infants has been studied and implemented in a range of settings [22,23]. The use of pentatonic lyres and harps combined with female humming or singing have shown to have positive impacts on infants' physiological signs and sleep states [6,24]. Schwilling et al investigated the effect of live pentatonic harp music on the infant's cortisol levels, and reported a decrease in cortisol levels as well as significantly reduced apneas, desaturations and bradycardia episodes [23]. Schlez et al [25] also found the use of live harp music had a significantly beneficial effect on maternal anxiety levels.

\section{Introduction of Harp Music in the NICU}

Given the indications of the beneficial effect of live harp music in infant regulation and maternal state, clinical observations were conducted of the effects of live improvisational harp music (LIHM) with a sample of neonates who were in the NICU for several weeks, and their families. The instrument used was a Reverie Harp (image Figure 1). The choice of this instrument was guided by the music therapist's previous experience in using the Reverie Harp with other clinical populations to assist with relaxation and pain management, and a clinical curiosity regarding its suitability and effectiveness in the NICU. The Reverie Harp has been 
specifically developed for use within a variety of therapeutic and health settings, ranging from NICUs to aged and palliative care $[26,27]$, however no studies in the use of this particular harp were found at the time of implementation. Studies using harp music in the NICU predominantly utilized precomposed songs or repetitive scales, with only one study implementing improvised harp music with no singing or humming [25]. There was no indication that the harp interventions previously studied were tailored and responsive to the infant. The series of clinical observations therefore provided opportunity to note the effects of LIHM tailored specifically to the infant's responses.

The Reverie Harp is a 22-string instrument, tuned to a pentatonic C scale (specifically named 'Reverie Tuning'). The middle two strings are both tuned to $\mathrm{C}$ (below middle $\mathrm{C}$ ), with ascending notes to the left (E, G, C, D, E, G, A, C, D, E) and right (G, A, C, D, E, G, A, C, D, G). The harp can be played sitting on a lap or cradled in the player's arms.

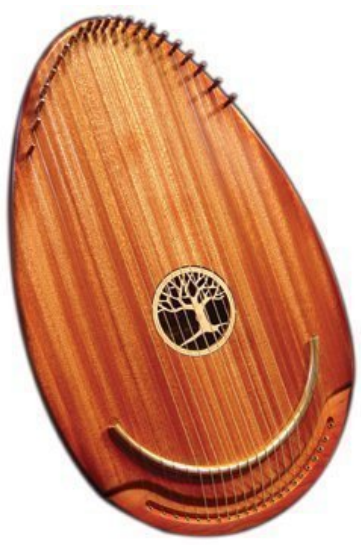

Figure 1: Reverie Harp

\section{Setting}

These clinical observations of LIHM were conducted in a surgical NICU that admits neonates with congenital or acquired abnormalities requiring surgery. Many of the neonates require several weeks or even months in hospital while their bodies recover and they transition to sucking feeds ready for discharge home. During the vital newborn period the brain growth is rapid (especially if born early) and the neonates are at risk of insults from stressors that they encounter while in the NICU [28]. This can include environmental stimulation from bright lights, noise, pain, procedures as well as variation in caregivers [29,30,31]. As part of the developmental care program [32] there has been a plan to introduce strategies to assist the neonates in coping with these stressors. One strategy has been to introduce quiet time (dedicated 3 hours) where there is an attempt to try to minimize the environmental stressors encountered so they have some protected time for sleep and interactions with their parents. Parents are also at risk of stress from having their infant in the NICU often for extended periods of time $[33,34,35]$.

\section{Implementation}

Infants that were in the NICU, born greater than 36 weeks gestation and were medically stable, were identified by the Clinical Nurse Consultant (NIDCAP Professional) and the parents were approached to participate. Four mothers and their infants were referred for the LIHM. The infants ranged from 36 weeks to 38 weeks gestation at birth and their ages ranged from one week to four weeks. This was the first MT intervention experienced by all parent-infant dyads. All the mothers were seated comfortably in a recliner chair and held their infant for all or most of the session that was carried out during Quiet Time between 1.30 and $2.30 \mathrm{pm}$. The staff was informed that the session was being held and were instructed to continue with any care the infant required.

The NIDCAP Professional used the NIDCAP naturalistic observation method where the infant is observed during an interaction or care-giving episode [36]. Behavioral observations using the NIDCAP methodology [37] were undertaken of the baby's response to the music in terms of their sleep states and cues for stress and self-regulation and coping strategies. The NIDCAP observation commenced by observing the infant's behavior before, during, and after intervention. Detailed observations of the infant's naturally occurring behaviors including autonomic, motor and attention/interaction were recorded. The music therapist arrived after the 10-20 minute pre-intervention observation, and made her own simultaneous observations of the infant that were recorded at the end of the session. At the commencement of the LIHM, the music therapist quietly sat beside the infant and mother, made a brief (sometimes nonverbal) greeting and began to play. In some cases, some brief dialogue between the mother and music therapist took place after the LIHM. The NIDCAP Professional remained approximately 10 minutes after the music for postintervention observation. Each session lasted in total between 45 and 60 minutes (including pre and post observation periods).

The clinical aims of the LIHM were to support infant regulation, and to enhance bonding and attachment between infant and parent during the unit's designated quiet time. The method chosen aligned with improvisational MT where live music is used to support state transition, as outlined by Shoemark [38]. The application of the LIHM was guided by the principle of 'entrainment' that Loewy [7] defines as involving the "application of live music elements catered to an infant's vital signs that influence the body's ability to regulate" (p.903). The musical elements (such as tempo and volume) were modified to match the infant's state to assist with stabilizing heart rate, avoiding overstimulation, and 
supporting attunement with the infant's parent. For example, if the infant's heart rate increased, the tempo would be slightly increased for a time to match this, and then gradually slowed whilst monitoring the infant's stabilizing heart rate. Any bodily responses of the infant (such as arm movements or facial expressions) were responded to with changes to rhythm and melody, often including slowing of tempo and slight pauses when the infant appeared to relax and settle.

As per recommendations for music used in the NICU [16], the harp was played with regular, rhythm between 40-50 beats per minute, with some slight fluctuations in response to the infant's state. Lower registers were also mainly used, and the strings were played with fleshy parts of fingers and thumb to create a smooth, soft timbre. Improvised single note melodies were played (4/4 meter), with occasionally two strings being played at once. Repetitive motifs would emerge from improvised melodies in an attempt to gently introduce the music and to 'hold and contain' the infant-parent dyad (examples Figure 2), This complied with recommendations to keep music simple, without complicated accompaniment to avoid overstimulation [16,39]. The music would be commenced with a simple motif (example Motif 1 \& 2, Figure 2 ), and modified accordingly in response to the infant, and at times the mother's breathing. In each case, the music was played continuously for 30-45 minutes.

Figure 2: Reverie Harp Motifs

Reverie Harp Motif I

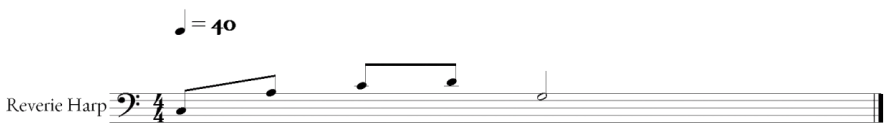

Reverie Harp Motif 2

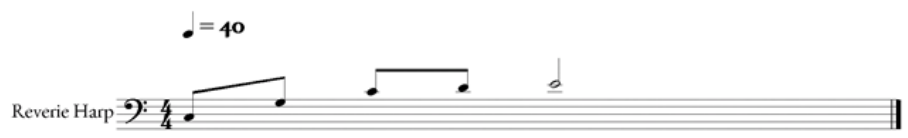

Reverie Harp Motif 3

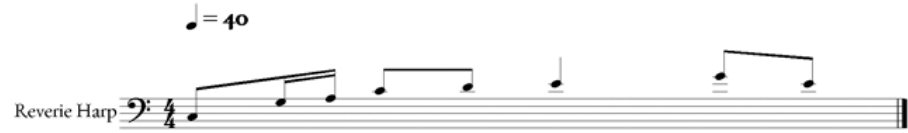

The following clinical vignettes are constructed from the NIDCAP nurse's observations and the music therapist's field notes. In each case, the story articulates the infant's prevailing condition and history, the position and state of the infant at the commencement of the session, descriptions of LIHM interventions and observed responses throughout, and ambient noise conditions in the NICU.

\section{Observations}

Case One.

Tony was born at 36 weeks gestation with a birth weight of 3410 grams. Following birth Tony was admitted to a special care nursery with respiratory distress before being transferred to the NICU for the management of cardiac failure due to a vein thrombosis. At the time of the MT session Tony was 2 weeks of age (38 weeks corrected) on demand breast-feeding and in the High Dependency section of the NICU. Just prior to the observation Tony and his mother had been to the ultrasound department for investigational scans and during this time he required a breast feed to settle.

Tony was observed for 70 minutes with 20 minutes being prior to the commencement of MT. His mother was holding Tony in her arms in the recliner chair next to his cot-space. Prior to the music commencing Tony had regular breathing at 40 - 64 breaths per minute, his heart rate ranged from 135 to 150 beats per minutes, he was pink with some mottling on his limbs. He was in an aroused state with flexed limbs and was able to transition into a light sleep for short periods despite some environmental noise of many voices, other babies crying, scraping trolleys, alarms and active computer keyboard use.

The music therapist played the Reverie Harp for approximately 45 minutes, seated one meter away from Tony and his mother. During the music session Tony remained in his mother's arms, initially he moved between a light robust sleep with periods of deep sleep. The ambient noise continued with crying and alarms. Simple, repetitive melodic motifs were played to provide a stable, 'holding' environment for Tony and his mother in the midst of the noise of the unit. The tempo and rhythmic structure of the music remained steady, and was only slightly varied to match any fluctuations in Tony's heart rate. The music therapist observed Tony's startle in response to the playing of a higher pitched string on the harp. Due to this, the music therapist played melodies in lower registers for the remainder of the session. Tony settled into a deep sleep together with his mother. His breathing was regular around 40 breaths per minutes and his heart rate was steady around 130 beats per minutes. He remained in a relaxed flexed position held against his mother's chest.

After the LIHM, Tony remained in a drowsy state with short periods of light and deep sleep. His breathing was regular around 50 breaths per minute and heart rate around $134 \mathrm{bpm}$. The ambience in the room changed as voices rose with people speaking loudly on the phone and the number of staff increased in the unit. Tony squirmed at times and placed his hands on his face and periodically frowned. 
Case Two.

Robert was born at 38 weeks gestation and weighed 3204 grams. He was admitted to the NICU at 8 days for management of his Pierre Robin Sequence and obstructive sleep apnea. At the time of his MT and observation he was receiving his feeds of expressed breast milk (EBM) from a squeeze bottle every four hours. His breathing was supported by continuous positive airway pressure (CPAP) at $8 \mathrm{cms}$ via a nasal mask when he was asleep in his cot. Robert was being prepared for discharge home on CPAP in a few days' time.

Robert was observed for 50 minutes that included 12 minutes prior to the music starting. His mother was holding him in an upright position on her chest in a recliner chair next to his cot. He was wrapped in a light sheet with his arms contained. He had just had a bottle-feed and had his diaper changed. His breathing was irregular and he was pink with occasional changes to red as he squirmed and moved around. He was sucking vigorously on a pacifier and was generally in a diffusely drowsy state with periods of being awake with fussy periods. His breathing was irregular around 52-60 breaths per minutes and his heart rate between $156-178 \mathrm{bpm}$.

As the music commenced Robert was cradled in his mother's arm supported by a small pillow. He was awake and sucking on his pacifier. He was squirming and his color became red with the effort. After 4-5mins of playing he settled for an extended time, before becoming unsettled again. He was re-wrapped with his hands free and he placed them on his face as he settled himself. Robert had short periods of light sleep and when in an aroused state he held onto his mother's finger and she interacted with a soft voice. His color settled to pink and he stayed in a flexed position with his hands on his face. The music therapist observed Robert appeared to settle with repetitive melodies on the harp that had more complex rhythm (example Motif 3 in Figure 2). When the melody was simplified and tempo slowed, this is when Robert would often move into unrest. The ambient noise was fairly muted with occasional voices in hushed tones. His mother commented at the end of the session that she thought Robert was relaxed, and it was the first time she had shared meaningful eye contact with Robert. She also commented that she felt relaxed, and thought this might help her express milk which she had to do next.

\section{Case Three.}

Angela was born at 38 weeks gestation and weighed 2700 grams. She was admitted to the NICU immediately following birth with a Gastroschisis for surgery. At the time of observation Angela was 3 weeks and 5 days of age. She was receiving total parenteral nutrition (TPN) via a central catheter and was on small trophic feeds of one $\mathrm{ml}$ of breast milk continuously via a gastric tube. She was being weaned off her morphine infusion that was used to manage her post- operative pain. Her report described her as irritable and she had commenced antibiotics for a high temperature.

Angela was observed for 40 minutes. Angela was initially held by her mother in a recliner chair and was put into her cot for the majority of the time for the LIHM. Angela's mother had to collect her other child who was brought into the nursery for the remainder of the session.

After 10 minutes, the music therapist sat and played Reverie harp for 30 minutes at the end of Angela's cot. Angela was pink and pale with episodes of irregular breathing between 43 - 68 breaths every minute. Her heart rate was regular between 142 - 168 bpm with oxygen saturation around 93-98\%. Angela was awake and went into short periods of drowsiness and light sleep. The music therapist observed Angela turning her head to track to the sound of the harp. She sucked on her pacifier as she moved her arms and legs in smooth erratic movements. Occasionally she would hold onto her tube and put her hands to her face. Her breathing during this time was irregular and fast. The music therapist used repetitive rhythms and melodies, only slightly modifying these when Angela roused. These musical modifications were used to match Angela's change of state, and repetitive melodic motifs were slowly re-introduced to assist Angela settle. The nursery environment was very noisy with many babies crying for extended periods and alarms ringing without being quickly cancelled. Angela appeared to struggle to calm herself during this time with the background noises. At times, it was difficult to hear the music. Angela's three-year-old brother came into the nursery with his mother during the music. He sat quietly next to his mother as she held Angela, spoke in a soft voice and appeared fascinated while looking at his sister and gently touching her.

\section{Case Four}

Jenny was born at 37 weeks gestation and weighed 2749 grams. She was admitted to the NICU at 23 days of age for management of her congenital hypoglycemia. At the time of the observation she was 5 weeks of age (42 weeks corrected). She was receiving EBM every four hours by tube given slowly over one hour. She was being tried with some sucking feeds. Her feed was due in one hour from when the music started.

Jenny was observed for 60 minutes including 12 minutes before music. Her room was very dark with some natural light filtering through the blinds. Jenny's mother was holding her in a recliner chair next to her cot. The sounds were soft on side of the nursery, however voices from a teaching session which was occurring across the room were constant. Jenny was in a flexed position in a soft wrap in her mother's arms. She was pink with irregular and fast breathing at $70-80$ breaths per minute. She was in a drowsy and sleepy state.

The music therapist sat opposite Jenny and her mother, playing LIHM for approximately 45 minutes. During the intervention Jenny was predominately in a light sleep with some periods of drowsiness and quietly awake. The music 
therapist commenced playing the harp with more complex melody and rhythms (using more semiquavers, with runs up and down strings), during which Jenny remained settled. Occasionally she would move slowly with a frown. This appeared to be in response to an alarm sounding in the room. When Jenny stirred, the music therapist played simpler, repetitive motifs to which Jenny settled quickly into. It was noted that her mother's breathing was in time with the tempo of harp melody. Jenny's breathing also settled into rhythmical pattern. Both mother and Jenny appeared to be in restful synchronicity throughout the session. Several times, the music therapist slowed and paused the music. Jenny consistently roused at this point, and then settled quickly once a steady rhythm was resumed. Her breathing was constant around 60 breaths and her oxygen saturation was steady around $92 \%$. Jenny looked relaxed and her mother appeared to drift to sleep. Her mother mentioned afterwards that her daughter's medical condition included a feature that she would respond well to rhythm. This knowledge came from the mother's own growing understanding and reading that she felt was confirmed by her daughter's response to the steady pulse and rhythms used.

\section{Discussion}

The utilization of LIHM appeared to be beneficial in responding to individual infants, and for entraining responses to help calm and regulate their behavior. In each case, the repetitiveness and/or complexity of melodic motifs and the tempo speed and variation were modified to match the infant's responses, and support their self-regulation. The differences in the musical elements played for each infant suggests no one piece of music is suitable for all and highlights the potential benefits of live music interventions that can respond and adapt to individual preferences and needs. In cases one and three, instances where the LIHM was modified to match a change in the infant's state are noted. This implies a musical communication whereby the music therapist acknowledges and responds to the infant. At times, the music therapist initiated the change in music out of clinical curiosity as to the level of entrainment, and to see if a reduction in tempo and melodic complexity would result in beneficial infant state changes such as deeper sleep. In several instances (cases Two and Four), these changes resulted in the infants becoming unsettled. The identification and investigation of possible factors behind this unintended consequence, such as the length of time repetitive motifs were played prior, the speed in which the modification of musical elements was made, and other environmental influences would be worth exploring in future studies. Another potential benefit of live music is nevertheless highlighted here in that the music therapist was able to immediately recognize an error in judgment and once again modify the intervention in response to the infant.

In addition to exploring the impacts of responsive live harp music interventions alone, further studies comparing the use of live versus recorded instrumental music is warranted. Several previous studies that have compared live and recorded singing have shown similar benefits of both types of music in comparison to no music conditions [40], as well as further effects of live music resulting in deeper sleep states $[6,40]$ and reduced heart rate [6]. As recorded instrumental music is commonly utilized in NICUs, there is value in investigating a comparison with live instrumental interventions to explore the importance of the responsiveness of musical elements in supporting infant regulation and facilitating bonding with caregivers.

The observations of infants and parents suggest the appropriateness of the Reverie Harp in this setting. Informal comments by staff and parents after LIHM often noted the relaxing nature of the music. The name of the harp reflects the intention of it's design to bring about a "a state of dreamy meditation or repose" [27]. This suggests the timbre of the harp elucidated associations with relaxation, calm and rest. The pentatonic scale may also be familiar across a range of cultures that would increase its applicability. The register of the harp was noted to have an impact on infant responses, with less stimulation observed in using the lower pitched strings on the harp. The music therapist avoided use of higher pitch strings when startle responses were observed, as in Case One. The use of other similar stringed instruments, such as guitar, may produce similar results in adhering to similar registers, melodic contours and rhythms, however these assumptions would require further investigation.

Due to an interest in observing the effectiveness of the Reverie Harp, it was decided to observe the effects of improvised pentatonic harp music alone without singing or humming. The use of infant directed singing and humming has clearly shown to have many benefits for infant behavioral states, neurodevelopmental stimulation, pacification, parentinfant attachment and promoting culturally sensitive, familycentered care $[7,12,14,16,19,20,40,41]$. It is anticipated that the inclusion of singing/humming interventions may have enhanced the effectiveness of MT in this setting, strengthened the therapeutic rapport with parents [21] and promoted the role of the parent and family in their infant's care, such as identifying 'song of kin' [19]. There is scope for further observation and comparison of the effectiveness of instrumental live music alone to interventions involving singing or humming (either accompanied or unaccompanied).

Differences were noticed in these cases between infants who were swaddled and held by their mother, and when they were lying in the cot. The observations of the infant's responses in Case Three who was in the cot for most the LIHM appeared more unsettled than for the infants being held. 
Previous studies have indicated the use of multimodal stimulation, touch and kangaroo care in conjunction with MT interventions to be of benefit $[12,22,24,25,41,42]$. This case points to the importance of considering the location of the infant, alongside types of MT interventions, in working towards therapeutic goals.

The potential for LIHM to reduce parental anxiety and to promote attachment and bonding is reflected in some of the observations and informal feedback given after the music intervention. The instance of first meaningful eye contact between mother and infant in Case Two and the observed synchronicity of breathing and music in Case Four serves as illustrations of how responsive LIHM may promote opportunities for bonding. Each of the mothers (and one father who observed one of the sessions) commented that they were themselves assisted to relax during the music sessions. Bieleninik et al's [2] meta-analysis identified three studies using live music combined with kangaroo care that demonstrated a reduction in maternal anxiety. It is recommended that including both mothers and fathers in measuring levels of bonding and attachment, and parental anxiety, be utilized in future research to test the effects of LIHM on these domains. In Case Four, it was noted with interest that the mother expressed her own knowledge of her daughter's condition in that she would respond to rhythm. Whilst the source of this information was not explored further, the mother's observations of her child's response to steady pulse and intricate rhythms supported her understanding. This implies the mother took an active role in the care and growing understanding of her child within the music intervention that is a key component of family-centered practice [21]. The interaction and behavior of the sibling in Case Three also highlights the potential role of LIHM in family-centered care as another avenue of future investigation.

The adverse impact of environmental noise on infants in the NICU is well documented $[16,43,44]$. The role of MT to modulate noise to reduce overstimulation has been explored and validated $[38,45]$. In this case, the extra noise in the room, particularly in Case Three, was observed to have varying impacts on the infants' responses. No measure was used for decibel levels, and would be warranted for future interventions especially given at times the harp was difficult to hear over other environmental sounds. Dearn and Shoemark's study emphasized the need for sufficiently quiet conditions to ensure music interventions are heard amongst the ambient environment to have meaningful effect [46]. This implies the choice of Reverie Harp may not have been suitable to be detected when ambient noise levels increase, and may be better suited to quieter conditions. This raises important considerations in choosing MT interventions that are of appropriate sound levels for the infants, suitable for the environment to assist with modulating adverse noise, and not leading to an increase of noise through louder staff discussion and activity $[38,44,46]$. Collaborating with NICU staff to ensure quiet conditions are maintained during interventions is imperative.

The behavioral observations and the responses to the LIHM were consistent with assisting the infants' selfregulation. Hanson-Abromeit [47] proposes NIDCAP provides a useful model to guide MT interventions as it emphasizes the importance of individualized interventions, balancing sensory stimulation to promote development, and parental involvement. Observing infants within the environment of the NICU before, during and following the LIHM using the NIDCAP approach enabled the observer to capture the positive effects of the LIHM on the infant's ability to settle and self-regulate. These observations were also useful in informing and supporting the music therapist's individualized, improvisational responses to each infant's cues. An example is in Case Two where the NIDCAP observer suggested re-wrapping the infant to allow his hands free to assist with self-regulation. The music therapist noted this, and modified her playing to find the rhythmic and melodic response to better assist the infant to settle afterwards.

These observations have demonstrated how a collaboration between music therapist and NIDCAP professional was beneficial to promote best outcomes for the infant, and would be recommended for future MT services. Professionals working together to identify infants who may benefit from LIHM has enabled an individualized program for vulnerable infants who are often overwhelmed by environmental stressors of the NICU.

\section{Conclusion}

The provision of LIHM by a qualified music therapist has been shown to be beneficial for medically stable neonates in the NICU. Using simultaneous observations by a certified NIDCAP Professional detailing the infant's natural behaviors in response to the music has provided a unique insight into the effect of LIHM. The observed responses of infants were consistent with self-regulation. The informal comments of parents also highlighted beneficial effects of relaxation for caregivers, and for meaningful moments of contact and bonding with their child. These clinical observations suggest scope for future research to confirm or refute the effects of LIHM on infants and parents. The Reverie Harp is a useful instrument in the delivery of LIHM, however may be only suited to quiet conditions to enable it to be detected within safe auditory limits. Using a music therapist in collaboration with an experienced nurse enables the identification of appropriate infants and their families who are likely to benefit, and is recommended for implementation of individualized MT services in the NICU. 


\section{Acknowledgements}

The babies and their families who participated in this observation, and the Cancer Centre for Children, The Children's Hospital at Westmead for supplying the Registered Music Therapist for the project.

\section{References}

1. Allen KA. Music therapy in the NICU: Is there evidence to support integration for procedural support? Adv Neo Care. 2013: 13(5): 349352.

2. Bieleninik L, Gold C. Early intervention for premature infants in neonatal intensive care unit. Acta Neuropsychologica. 2014: 12(2): 185203.

3. Haslbeck FB. Music therapy for premature infants and their parents: An integrative review. Nord J Mus Therapy. 2012: 21(3): 203-226.

4. Pölkki T, Korhonen A. The effectiveness of music on pain among preterm infants in the NICU: A systematic review. JBI Database of Systematic Reviews and Implementation Reports. 2014: 12(4): 354-373.

5. Standley JM. Music therapy research in the NICU: An updated metaanalysis. Neo Netw. 2012: 31(5): 311-316.

6. Arnon S, Shapsa A, Froman L, et al. Live music is beneficial to preterm infants in the neonatal intensive care unit environment. Birth. 2006: 33: $131-136$.

7. Loewy J, Stewart K, Dassler AM, et al. The effects of music therapy on vital signs, feeding, and sleep in premature infants. Pediatrics. 2013: 131(5): 902-918.

8. Badr LK, Demerjian T, Daaboul T, et al. Preterm infants exhibited less pain during a heel stick when they were played the same music their mothers listened to during pregnancy. Acta Pædiatrica. 2017: 106: 438445.

9. Bergomi P, Chieppi M, Maini A, et al. Nonpharmacological techniques to reduce pain in preterm infants who receive heel-lance procedure: A randomized controlled trial. Res Theory Nurs Pract: Int J. 2014: 28(4): 335-348.

10. Butt ML, Kisilevsky BS. Music modulates behavior of premature infants following heel lance. Canadian J of Nurs Res. 2000: 31: 17-39.

11. Keith DR, Russell K, Weaver BS. The effects of music listening on inconsolable crying in premature infants. J Mus Ther. 2009: 46: 191203.

12. Malloch S, Shoemark H, Crncec R, et al. Music therapy with hospitalized infants-the art and science of communicative musicality. Infant Mental Health J. 2012: 33(4): 386-399.

13. Cevasco A, Grant R. Effects of the pacifier activated lullaby on weight gain of premature infants. J Mus Ther. 2005: 42: 123-139.

14. Ettenberger $\mathrm{M}$, Cárdenas $\mathrm{C}$, Parker $\mathrm{M}$, et al. Family-centred music therapy with preterm infants and their parents in the Neonatal Intensive Care Unit (NICU) in Colombia - A mixed-methods study [published online 21 July 2016]. Nord J Music Therapy. doi: 10.1080/08098131.2016.1205650.

15. Standley JM, Cassidy, J, Grant, R, et al. The effect of music reinforcement for non-nutritive sucking on nipple feeding of premature infants. Pediatr Nurs. 2010: 36(3): 138-145.

16. Shoemark H, Hanson-Abromeit D, Stewart L. Constructing optimal experience for the hospitalized newborn through neuro-based music therapy. Frontiers in Human Neuroscience. 2015: 9: 487: 1-5.

17. Walworth D, Standley JM, Robertson A, et al. Effects of neurodevelopmental stimulation on premature infants in neonatal intensive care: Randomized controlled trial. J Neon Nurs. 2012: 18: 210216.
18. Lai HL, Chen CJ, Peng TC, et al. Randomized controlled trial of music during kangaroo care on maternal state anxiety and preterm infants' responses. Int J Nurs Stud. 2006: 43(2): 139-146.

19. Loewy J. NICU music therapy: Song of kin as critical lullaby in research and practice. Annals of New York Academy of Science. 2015: 1337: 178185.

20. Haslbeck, FB. The interactive potential of creative music therapy with premature infants and their parents: A qualitative analysis. Nord J Music Therapy. 2014: 23(1): 36-70.

21. Shoemark H, Dearn T. Keeping parents at the centre of family centred music therapy with hospitalised infants. Aust J Music Ther. 2008: 19: 324.

22. Henning I. Music therapy with premature infants: Insights and recommendations from the current literature and a German pilot project. Canadian J Music Therapy. 2012: 18(1): 26-44.

23. Schwilling D, Vogeser M, Kirchhoff F, et al. Live music reduces stress levels in very low-birthweight infants. Acta Pædiatrica, Int J Paeds. 2015: 104(4): 360-367.

24. Teckenberg-Jansson P, Huotilainen M, Pölkki T, et al. Rapid effects of neonatal music therapy combined with kangaroo care on prematurelyborn infants. Nord J Music Therapy. 2011: 20(1): 22-42.

25. Schlez A, Litmanovitz I, Bauer S, et al. Combining kangaroo care and live harp music therapy in the neonatal intensive care unit setting. Israel Med Assoc J. 2011: 13(6): 354-358.

26. Roberts Music Australia, Reverie Harps. Available at: http://www.robertsmusic.net/harps/. Accessed 30 January 2017.

27. Music Makers, The Reverie Harp. Available at: http://www.harpkit.com/category/revharp.html. Accessed 30 January 2017.

28. Kolb B, Gibb R. Brain plasticity and behaviour in the developing brain. J Can Acad Child Adolesc Psych. 2011: 20(4): 265-276.

29. Harrison D, Bueno M, Reszel J. Prevention and management of pain and stress in the neonate. Res Rep Neo. 2015: 5: 9-16.

30. Maschke C, Rupp T, Hecht K. The influence of stressors on biochemical reactions - a review of present scientific findings with noise. Int J Hyg Environ Health. 2000: 203: 45-53.

31. Wachman EL, Lahav A. The effects of noise on preterm infants in the NICU. Archives of Disease in Childhood - Fetal and Neonatal Edition. 2011: 96: F305-F309.

32. Spence K. Seeking the evidence for a system change in a surgical NICU. Dev Observ. 2012: 5(2): 16-19.

33. Barr P. A dyadic analysis of negative emotion personality predisposition effects with psychological distress in neonatal intensive care unit parents. Psychol Trau: Theory, Res, Pract Policy. 2012: 4(4): 347-355.

34. Franck L, Cox S, Allen A, et al. Measuring neonatal intensive care unitrelated parental stress. J Adv Nurs. 2005: 49(6): 608-615.

35. Busse M, Stromgren K, Thorngate L, et al. Parent responses to stress: In the neonatal intensive care unit. Crit Care Nurs. 2013: 33(4): 52-60.

36. Lawhon G, Hedlund R. Newborn Individualized Developmental Care and Assessment Program Training and Education. J Perinat Neonat Nurs. 2008: 22(2): 133-144.

37. Als H. Newborn Individualized Developmental Care and Assessment Program (NIDCAP): new frontier for neonatal and perinatal medicine. J Neo-Peri Med. 2009: 2: 135-147.

38. Shoemark H. Working with full-term hospitalized infants. In: Bradt J. ed. Guidelines for Music Therapy Practice: Pediatric Care. Philadelphia, PA: Barcelona Publishers; 2013: 116-151.

39. Standley J M, Walworth D. Music therapy with premature infants: Research and developmental interventions. Silver Spring, MD: American Music Therapy Association; 2010.

40. Garunkstiene R, Buinauskiene J, Uloziene I, et al. Controlled trial of live versus recorded lullabies in preterm infants. Nord J Music Therapy. 2014: 23(1): 71-88. 
41. Shoemark H. Singing as the foundation for multi-modal stimulation. In Pratt R, Grocke D, eds. MusicMedicine3, MusicMedicine and Music Therapy: Expanding Horizons. Victoria, Australia: The University of Melbourne. 1999: 140-152.

42. Standley JM. The effect of music and multimodal stimulation on responses of premature infants in neonatal intensive care. Pediatr Nurs. 1998: 24(6): 532(1-9).

43. Graven SN, Sound and the developing infant in the NICU: Conclusions and recommendations for care. J Perinatology. 2000: 20(8): S88-93.

44. Browne JV. Developmental care for high-risk newborns: Emerging science, clinical application, and continuity from newborn intensive care unit to community. Clin Perinat. 2011: 38: 719-729.

45. Standley JM, Whipple J. Music therapy for premature infants in the neonatal intensive care unit: Health and developmental benefits. In S L Robb, ed, Music therapy in pediatric healthcare: Research and evidenced-based practice. Silver Spring, MD: American Music Therapy Association; 2003: 19-30.

46. Dearn T, Shoemark, $H$. The effect of maternal presence on premature infant responses to recorded music. J Obstet Gynecol Neonatal Nurs. 2014: 43: 341-350.
47. Hanson Abromeit D. The Newborn Individualized Developmental Care and Assessment Program (NIDCAP) as a model for clinical music therapy interventions with premature infants. Music Therapy Perspectives. 2003: 21: 60-68.

\section{Biographical Statements}

Roxanne McLeod BSocSc, MAMusTher, Registered Music Therapist. Employed in hospital and community settings, specializing in pediatrics and early intervention. Child Life and Music Therapy Department, Cancer Centre for Children, The Children's Hospital at Westmead.

Kaye Spence AM, RN, MN. Clinical Nurse Consultant Neonatology. An experienced neonatal nurse, clinical researcher and certified NIDCAP professional. Grace Centre for Newborn Care, The Children's Hospital at Westmead, Western Sydney University 